$\xi=1$ 圆

\title{
Electrical and Protrusion Stimulation System for Braille Recognition Training
}

\author{
Hakyeong Jeong, Myungin Hwang ${ }^{2}$, Hojun Yeom $^{3 *}$ \\ ${ }^{1}$ Eulji University, Seongnam, Korea \\ * Corresponding author E-mail:hyeom@eulji.ac.kr
}

\begin{abstract}
As the number of blind people increases due to acquired factors, the necessity of a device capable of recognizing the braille quickly and accurately has been increased, and research is underway to develop a braille device for enhancing the readability of the braille. This study suggests a system to increase the recognition rate of braille by fusing visual information through tongue electrical stimulation device and Braille output device based on the protruding tactile sensation based on the method of applying electrical stimulation to the tongue. In particular, considering Korean Braille characteristics and Braille recognition error, we propose a system to increase the Braille resolution by separating the consonants into protruded Braille output devices of servo motor type and Braille output device of electrical stimulation type.
\end{abstract}

Keywords: App Inventor; Braille; Electrical stimulation; Servo motor; STM32F407;

\section{Introduction}

Since the end of the 20th century, however, the importance of the information to maximize the information revolution with the introduction of the society based on knowledge and information rapidly as information society, said the development of the digital society. In recent years, such as the electronic devices such as a Smartphone e-book from reading through the news until all the information is easily accessible [1]. The convenience of normal people is universalized, while the access of information to the disabled is stagnant compared to this development. Blind people should learn Braille to replace letters. For the visually impaired, braille has more meaning than letters, and has confidence and independence. Visually impaired persons who can read and write Braille well have higher employment rate and higher self-esteem than visually impaired people who do not know braille [2]. Braille is a necessary means to transform blind people into socially active talent. The process of learning braille is an essential process for the blind to live as a social person. Despite this, visually impaired people who do not know braille account for a majority of the total visually impaired population. The reason is that the learning speed of the blind is so slow that the learning motivation can easily be frustrated and the braille learning process is not interesting. And one of the difficulties in teaching visually impaired learners is that they are much slower than non - disabled learners.

Another issue is the size and cost of the braille book. Because of the feature of Korean braille, braille book is usually large and thick, and it is difficult to make it because it does not have many users in comparison with the labor required to take with braille typewriter without inking for production. Therefore, there is a need for tools to assist in information acquisition and braille learning.

Braille is a letter designed to allow the visually impaired to read with the tactile sense instead of the printed letter so that it can be read using time. In the past, people with visual impairments used braille books to acquire information, but as we move into the information age, there are a number of machines that can acquire information in addition to books, so we are quickly acquiring the necessary information beyond time and space constraints. Braille electronic devices are being developed to help people with visual impairments to acquire information [1].

The types of research related to braille electronics are divided into three categories. First, there is research to restore the visual function of the visually impaired through the visual substitution device. The black and white image obtained through the camera is electrically stimulated in the visual cortex of the occipital lid, thereby confirming the success of the clinical test that the visually impaired can accept the visual information and actively move.

Insert the electrode array into the visual cortex of the occipital lobe as shown in figure 1. The image projected on the CCD camera mounted on the visual aid is digitized to create a monochrome display on the electrode array. The electrode produces a visual phenomenon caused by the pressure applied to the eyeball without any stimulation by one to four densely conscious light senses, that is, it can recognize the monochrome display.

In addition to the method of inserting the electrode array directly into the visual cortex, visual information can be obtained by electrically stimulating the tongue electrode array. Animal experiments and clinical trials have revealed that both congenital and acquired visual impairments can process and interpret visual association information in other senses than in the eye [3].

Grant et al. (2016) conducted a study to evaluate the functional usability of visual information obtained through electrical stimulation of the tongue and electrical stimulation safety in clinical trials [4]. Using the visual information displayed on the tongue, we were able to perform the search for obstacles successfully in the natural environment much more consistently than other blind people. In clinical trials, $91.2 \%$ of the subjects were able to identify and touch more than nine of the 20 objects successfully, and $57.9 \%$ of the subjects were able to read more than six words correctly. 
The second is to develop a dynamic braille cell that provides a tactile display to reduce the spatial waste of writing characters. The Korean braille character is composed of the primitive, neutral, and consonant words, which are used to generate a complete character, which requires three braille codes. In a study using electrical stimulation to implement a dynamic braille cell, we found that tactile sensations obtained through electrical stimulation accept sensations similar to physical stimuli. In addition, in a study related to a system that applies electrical stimulation by applying a pulse to the tip of a finger, the recognition rate of the braille becomes higher as the stimulation pulse is applied by touching with the fingertip, and the recognition time of the electrical stimulus becomes shorter and shorter Recognition has been derived. Next, studies are being conducted to realize a solenoid-type dynamic braille cell. The solenoid method is cheap, and it can be operated easily by applying only the required current and voltage, but it is difficult to miniaturize, and it is difficult to read the braille because it is impossible to set the time difference and the degree of protrusion of the braille cell.

On the other hand, the servo motor method has a merit that the degree of protrusion of the braille cell can be easily determined by finely adjusting the angle, and the control method is simple by the PWM method. In addition, it has been investigated that a device implemented by a servomotor can help to speed up the reading of braille .

Thirdly, studies are being conducted to investigate the degree of recognition of the braille error and to suggest directions to improve the readability of the braille. As a result of analyzing the types of errors in the use of braille for the visually impaired, the visually impaired showed a lot of errors in the braille when displaying the braille. Also, when reading braille characters, there were many cases where they missed the lyric sounds, diphthongs, and abbreviations. In order to increase the recognition rate of braille, it is considered that the ability to understand the sentence will be enhanced by allowing the braille cell to read the braille, consonant, abbreviation and abbreviation in different ways. In the previous study, if we discussed the passive reading using a fixed braille output device, this study fused a dynamic single braille cell with an electric stimulus braille cell.

It will distinguish consonants, vowels, and consonants considering Korean braille characteristics, which will make it more suitable for Koreans, enable fast and accurate braille recognition, and recognize the visual information together through the tongue stimulation device, which will result in braille recognition and effective training.

\section{Methods}

The system can be divided into a sensor unit, a control unit, and a driving unit. The electric stimulator and the braille output device are controlled by transferring the data to the MCU by creating an app that separates the input words that wanted to output into braille using App Inventor into the initial, medial, and final consonant.
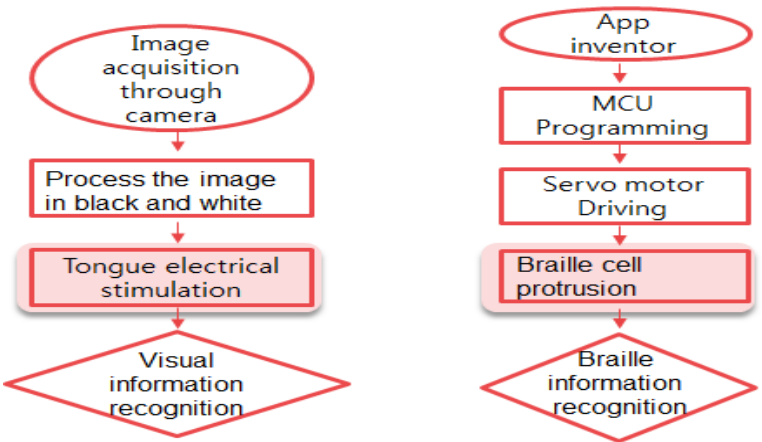

Fig. 1: Algorithm electrical and protrusion stimulation system for braille recognition training

\subsection{The Sensor Unit}

We programmed an app that uses MIT App Inventor 2 to separate words into hyphens, neutrals, and vertices to match the alphabetical arrangement of the keyboard. In the firmware program we used, Korean language was not recognized, so Korean and English were replaced by keyboard, and the consonants were differentiated by capital and lower case.

\subsection{The Control Unit}

In order to generate the protrusion by operating the servo motor according to the data received through the Bluetooth, a control unit is required. In this study, MCU, which is the core of this control, was selected by STM32F407VG. This MCU is a DSPcapable 32bit microcontroller with multiple interrupt processing, and has many peripherals. One of them, the UART, is a peripheral device required to communicate with the App Inventor. Another peripheral device, PWM(pulse width modulation) of the timer, was used to adjust the angle of the servomotor according to the braille and adjust the intensity of the electric stimulus. Recognition time for electric stimulation can be recognized in a short time according to the number of experiments. Since it takes 10 seconds to recognize one character, the time delay between the servo motor and the electric stimulus braille cell is set to 3 seconds. A Bluetooth module called HC-06 was used to transmit the contents of the app inventory to the MCU.

\subsection{The Stimulation Unit}

The character information captured in the app has developed a tongue electrical stimulation device that gives the tongue, which is ideal for the perception of the senses, close to nerve fibers closer to other parts of the body, making electrical impulses and tiny vibrations. Through sensory stimulation using electrical stimulation, visual information recognized through tongue and tactile information of braille output device are fused to enable accurate braille recognition. The tongue electrical stimulation device is composed of six stainless steel electrodes arranged in a $2 \times 3$ grid and has a pin configuration, and character information is output according to image information according to electrical stimulation intensity. An electric stimulation circuit with an output voltage of $1.414 \mathrm{Vrms}$ was designed by applying a $20 \mathrm{kHz}$ pulse frequency to recognize character information on the tongue through electrical stimulation. PWM control through timer of MCU is implemented. PWM1 is implemented to apply a $90 \%$ duty ratio and $10 \mathrm{~Hz}$ with $3.3 \mathrm{~V}$ size, and PWM2 to apply $20 \mathrm{kHz}$ with a $50 \%$ duty ratio and $3.3 \mathrm{~V}$ size.

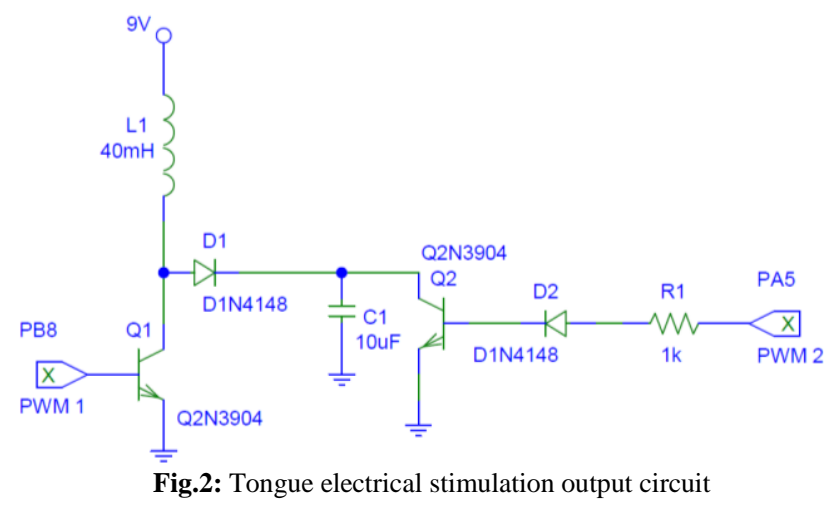

As shown in the figure $\mathrm{xx}$, the developed braille output device consists of 6 pins to represent braille composed of $2 \times 3$, and a mechanism for projecting the braille pin to rotate by a specific angle using a servo motor and $6 \times 3$ array It is developed to be an efficient braille recognition device by fusing a mechanism that 
recognizes the braille to output by giving electrical stimulus to the needle electrode.

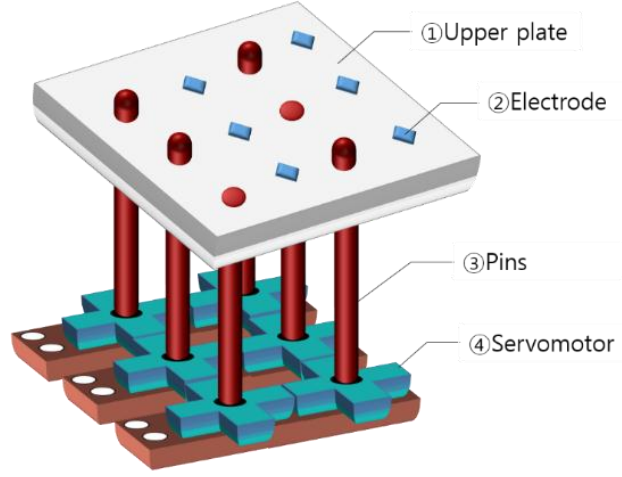

Fig. 3: The protruded braille cell output section

The mechanism for projecting the braille pins was the SG-90 micro servomotor. Since it moves between $-90 \sim 90^{\circ}$, the position of pin can be controlled by PWM control method and the degree of protrusion of pin can be adjusted. In order to output the consonant, the servo motor was operated by $20^{\circ}$ to realize a protrusion of about $1.5 \mathrm{~cm}$. In addition, the interval between the braille cells is about $6 \mathrm{~cm}$ in width and about $1 \mathrm{~cm}$ in length, and a delay time of about 3 to 4 seconds is set by coding the delay. In order to recognize the braille by applying the electric stimulus, the contact portion is important because the voltage according to the electric energy changes according to the area of the contact portion. A copper needle electrode was used as the material of the contact part, and the output voltage was about $60 \mathrm{~V}$ in consideration of the voltage recognized by the finger. The output was composed of 6 channels of $2 \times 3$ array, and vowels with low recognition rate were output.

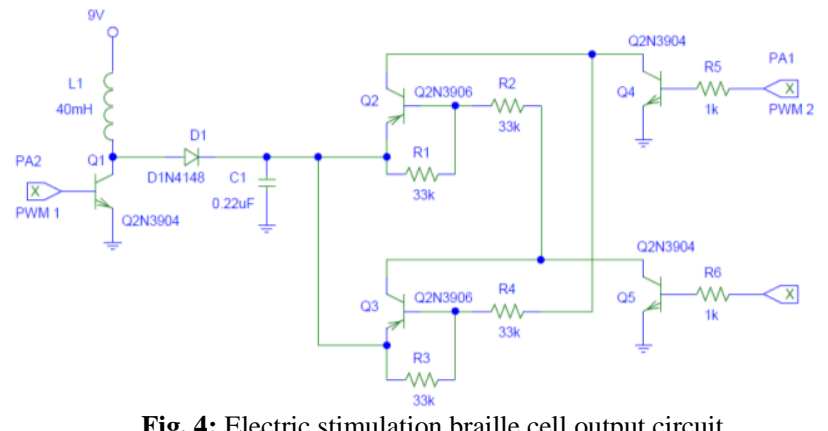

Fig. 4: Electric stimulation braille cell output circuit

\section{Results}

Figure 5 shows that the output of the tongue electrical stimulator is normal. Vout is the output of $1.36 \mathrm{~V}$ which is close to $1.414 \mathrm{~V}$.

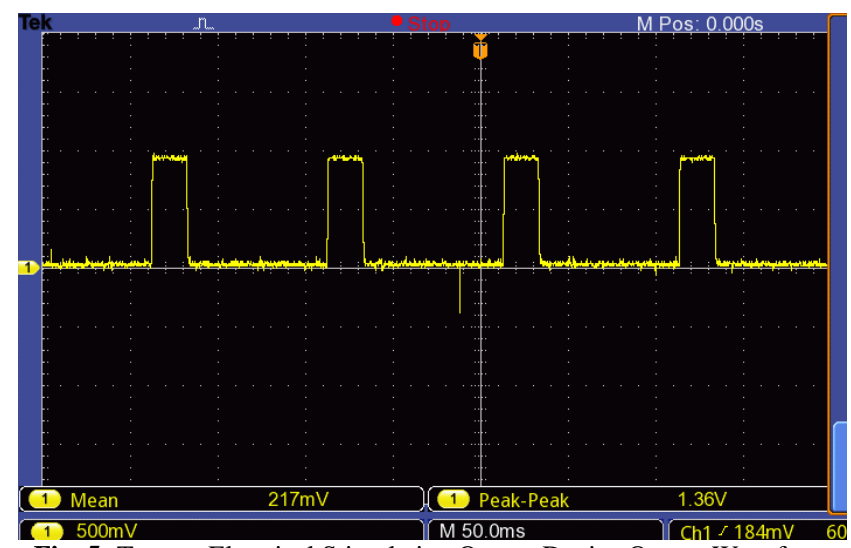

Fig. 5: Tongue Electrical Stimulation Output Device Output Waveform
After initializing 6 servo motors with MCU, braille cell protruded when voltage is applied. When the voltage is applied, the servo motor operates at a preset angle and protrudes and returns to the initial value. As a result of measuring the signal applied oscilloscope, it was confirmed that the waveform of the PWM control method appears.

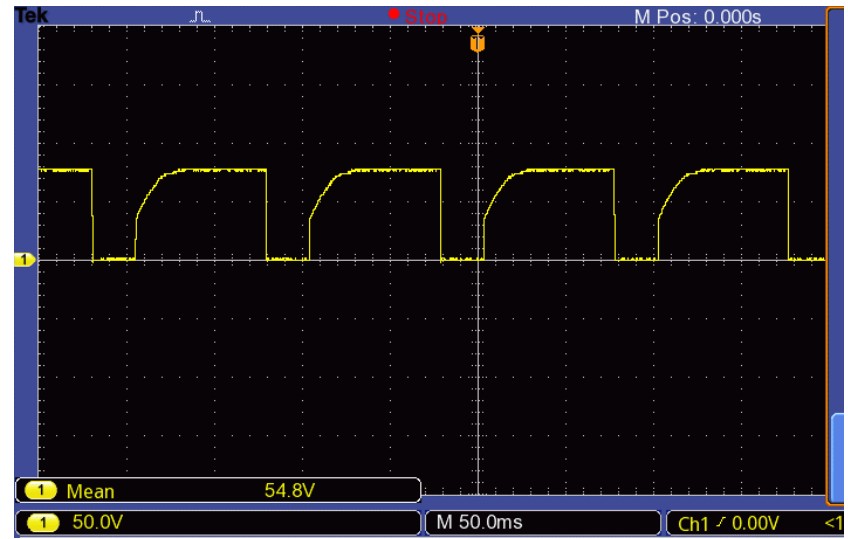

Fig. 6: Electrical stimulation braille cell output device waveform

\section{Conclusion}

In this study, visual information was recognized on the tongue rather than eyes for the visually impaired by using the tongue electrical stimulation device, while the consonant output the braille using the protruded braille cell, whereas the braille cell We developed a bi - directional braille output device which is convenient for blind people and can easily recognize braille at the same time as conventional unidirectional braille output device.

In this study, it was found that only $2 \%$ of the best-selling books were published as braille books, and the e-book and the study, The cultural complaint of the visually impaired will be resolved if the visually impaired person freely reads the e-book.

\section{Acknowledgement}

This paper was suported by Eulji University in 2018 .

\section{References}

[1] Jeong-ki Hong et. al, “ The Interface of Wearable Electronic Braille Device", HCI 2014, pp.17-20, Feb. 2014

[2] Maurice P., Ron K.," "The tongue as a portal to the visual cortex in congenital blindness: the blind's brain", Oct. 2011

[3] Kupers, R., Pietrini, P., Ricciardi, E. and Ptito, M, "The nature of consciousness in the visually deprived brain Frontiers in Psychology", Vol.2, No.19, pp.1-15, Feb. 2011

[4] Patricia Grant, 2016, "The Functional Performance of the BrainPort V100 Device in Persons Who Are Profoundly Blind", Journal of Visual Impairment \& Blindness Cho JH, Chang SA, Kwon HS, Choi YH, KoSH, Moon SD, Yoo SJ, Song KH, Son HS, Kim HS, Lee WC, Cha BY, Son HY \& Yoon KH (2006), Long-term effect of the internet-based glucose monitoring system on HbA1c Reduction and glucose stability: a 30-month follow-up study for diabetes management with a ubiquitous medical care system. Diabetes Care $29,2625-2631$. 